\title{
Aspectos epidemiológicos das notificações de malária no Piauí
}

\author{
Epidemiological aspects of malaria notifications in Piauí \\ Aspectos epidemiológicos de las notificaciones de malaria en Piauí
}

Recebido: 18/04/2021 | Revisado: 26/04/2021 | Aceito: 29/04/2021 | Publicado: 14/05/2021

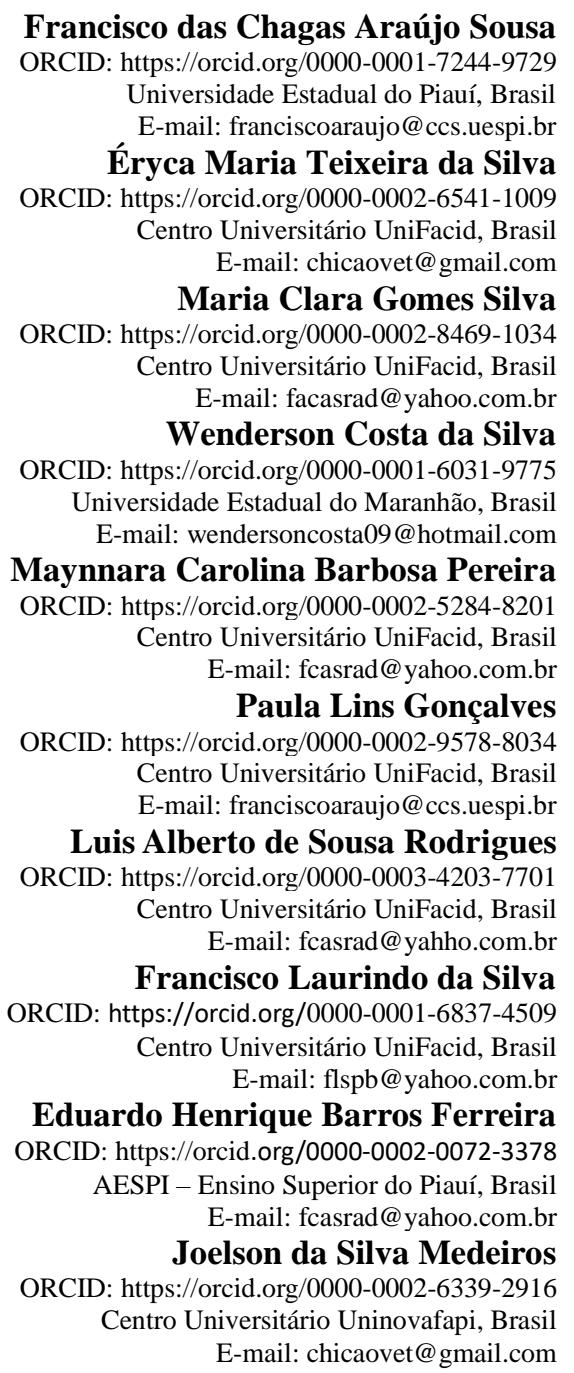

\section{Resumo}

A malária é uma doença infecciosa parasitária, de grande impacto na saúde mundial, causada por parasitas do gênero Plasmodium. É transmitida através de um vetor, a fêmea infectada do mosquito Anopheles ou popularmente conhecido por mosquito prego, logo, não é uma doença contagiosa não sendo transmitida de pessoa para pessoa. Este estudo teve como objetivo descrever a situação epidemiológica da malária no Piauí entre os anos de 2015 e 2019. Realizou-se um levantamento epidemiológico dos casos de malária no Piauí entre os anos de 2015 a 2019 através das plataformas SINAN-DATASUS, utilizando as variáveis sexo, faixa etária, raça, espécie do protozoário e municípios com notificação. Os resultados encontrados foram expressos em tabelas do Excel. Observou-se que as cidades com maior quantidade de casos foram Luzilândia e Teresina, além disso, a doença propagou-se mais entre os pardos e em homens na faixa dos 20-39 anos. Ademais, a espécie mais encontrada nos resultados parasitológicos foi o Plasmodium falciparum. O Piauí não apresenta uma quantidade significativa de casos, mas ainda é necessário reforçar a vigilância e controle e prevenção de casos através de medidas rápidas e efetivas.

Palavras-chave: Epidemiologia; Malária; Saúde pública; Sistemas de informação em saúde; Vigilância em saúde pública. 


\begin{abstract}
Malaria is a parasitic infectious disease, with a great impact on global health, caused by parasites of the genus Plasmodium. It is transmitted through a vector, the female infected with the Anopheles mosquito or popularly known as the nail mosquito, therefore, it is not a contagious disease and is not transmitted from person to person. This study aimed to describe the epidemiological situation of malaria in Piauí between the years 2015 and 2019. An epidemiological survey of malaria cases in Piauí was carried out between the years 2015 to 2019 through the SINANDATASUS platforms, using the variables sex, age group, race, protozoan species and municipalities with notification. The results found were expressed in Excel tables. It was observed that the cities with the highest number of cases were Luzilândia and Teresina, in addition, the disease spread more among browns and in men aged 20-39 years. Furthermore, the species most found in the parasitological results was Plasmodium falciparum. Piauí does not have a significant number of cases, but it is still necessary to strengthen surveillance and control and prevention of cases through rapid and effective measures.
\end{abstract}

Keywords: Epidemiology; Malaria; Public health; Health information systems; Public health surveillance.

\title{
Resumen
}

La malaria es una enfermedad infecciosa parasitaria, de gran impacto en la salud mundial, causada por parásitos del género Plasmodium. Se transmite a través de un vector, la hembra infectada con el mosquito Anopheles o popularmente conocido como mosquito de las uñas, por lo tanto, no es una enfermedad contagiosa y no se transmite de persona a persona. Este estudio tuvo como objetivo describir la situación epidemiológica de la malaria en Piauí entre los años 2015 y 2019. Se realizó una encuesta epidemiológica de casos de malaria en Piauí entre los años 2015 a 2019 a través de las plataformas SINAN-DATASUS, utilizando las variables sexo, grupo de edad, raza, especie del protozoo y municipios con notificación. Los resultados encontrados se expresaron en tablas de Excel. Se observó que las ciudades con mayor número de casos fueron Luzilândia y Teresina, además, la enfermedad se extendió más entre marrones y en hombres de 20 a 39 años. Además, la especie más encontrada en los resultados parasitológicos fue Plasmodium falciparum. Piauí no tiene un número significativo de casos, pero aún es necesario fortalecer la vigilancia y el control y la prevención de casos a través de medidas rápidas y efectivas.

Palabras clave: Epidemiología; Malaria; Salud pública; Sistemas de información en salud; Vigilancia en salud pública.

\section{Introdução}

A malária é uma doença infecciosa parasitária, de grande impacto na saúde mundial, causada por parasitas do gênero Plasmodium. É transmitida através de um vetor, a fêmea infectada do mosquito Anopheles ou popularmente conhecido por mosquito prego, logo, não é uma doença contagiosa não sendo transmitida de pessoa para pessoa. É uma enfermidade com sintomas pouco específicos que incluem febre alta simultânea a calafrios, sudorese intensa e cefaleia. É comum sua ocorrência em regiões tropicais e sua incidência está interligada aos fatores sociais, econômicos, étnicos/raciais e culturais (Gomes et al., 2020).

A patogenia da malária é caracterizada pela febre terçã e quartã, sua evolução pode ser aguda ou crônica e o ciclo de vida do parasita determina a evolução da doença. O ciclo inicia com a picada do mosquito infectado que transmite o Plasmodium na forma de esporozoíto e a partir daí inicia-se a multiplicação no organismo humano. Em seguida, podem surgir os sintomas entre e 15 dias após a picada do vetor (febre alta simultânea a calafrios, sudorese intensa e cefaleia, etc.) o que caracteriza o período de incubação, e por serem comuns em outras doenças dificulta-se o diagnóstico clínico (Do Nascimento, 2015).

A malária é uma doença curável, no momento, em que o paciente testa positivo e o tratamento é iniciado. O Sistema Único de Saúde (SUS) fornece medicamentos que auxiliam no combate ao protozoário, quando há casos mais graves o paciente é internado imediatamente. Contudo, a cura só é garantida se a detecção e tratamento forem iniciados precocemente. Após a recuperação, o paciente deve realizar testes para controle de cura (Ministério da Saúde [MS], 2019).

No Brasil, cerca de $99 \%$ dos casos de malária centralizam-se na região Amazônica composta por 9 estados (Acre, Amapá, Amazonas, Maranhão, Mato Grosso, Pará, Rondônia, Roraima e Tocantins). O cenário da malária atualmente indica 
que existe, além da região endêmica descrita anteriormente, outras áreas de focos da doença. Cerca de 1\% dos casos de malária no Brasil, ocorrem na região extra-amazônica e são ocasionados por presença de pessoas contaminadas vindas de regiões da Amazônia ou, até mesmo, da África e Ásia. É evidente que, a espécie do parasita muda conforme a região de transmissão. Nos últimos anos as espécies de maior ocorrência foram o P. falciparum e o $P$. vivax nas regiões do Brasil (MS, 2010).

A OMS relata que a ocorrência de malária é favorecida pelos fatores climáticos (precipitação pluviométrica, umidade relativa do ar e temperatura) e por isso, é comum na região amazônica. Nessa região, a malária pode ocasionar anemia grave nos pacientes. Os estudos relatam que a forma mais letal da doença é ocasionada pelo P. falciparum (MS, 2020).

O Piauí por fazer fronteira com o Maranhão, englobado pela região Amazônica, sempre permanece alerta em relação ao número de casos de malária. Mesmo que seja uma doença pouco comum no local, as equipes de saúde mostram-se sempre preparadas para vigilância e notificação de casos da enfermidade. Apenas em meados de 1970 e 1986, que a doença foi preocupante na região piauiense, mas com as ações estabelecidas os casos foram controlados. Grande parte dos casos notificados no Piauí são de outros lugares, que buscam atendimento no estado (Fonseca, 2017; Secretária de Saúde do Estado do Piauí [SESAPI], 2017).

Apesar de o Piauí possuir uma localização próxima a região endêmica, clima e perfil sociodemográfico favoráveis ao surgimento da malária, a situação da doença no estado tem sido pouco abordada nos últimos anos. Nessa perspectiva, o objetivo do presente trabalho foi descrever a situação epidemiológica da malária no Piauí entre os anos de 2015 e 2019.

\section{Metodologia}

Trata-se de um estudo descritivo, quantitativo e transversal das notificações de malária no estado do Piauí entre os anos de 2015 a 2019, em que foram analisados os dados secundários de malária disponíveis no do Departamento de Informática do SUS (DATASUS) do Sistema de Informação de Agravo de Notificações (SINAN).

Os critérios de inclusão foram todos os casos de malária no Estado do Piauí, no período de 2015 a 2019, constantes da base de dados DATASUS do Ministério da Saúde, inseridos no programa SINAN. Os critérios de exclusão foram os casos notificados fora do Estado supracitado, que não constem do SINAN, além daqueles fora do recorte temporal.

Foram analisadas as seguintes variáveis: número de casos de malária, características das ocorrências dos casos de malária segundo a espécie parasitária, raça mais atingida, faixa etária, morbidade, sexo e a distribuição das notificações de casos confirmados cidades do agravo. Os dados coletados foram apresentados em gráficos projetados no Excel 2010, para análise epidemiológica de maior relevância.

\section{Resultados}

Dentro do período avaliado, de 2015 a 2019, foram notificados um total 95 casos de malária no estado do Piauí. De acordo com a Figura 1, o ano de 2015 obteve o menor número de casos com 15 (15,79\%) notificações. O ano de 2016 atingiu o maior número de casos no Piauí $(23,16 \%)$. Diante disso, observou-se que nos anos analisados houve variações nos números de ocorrências. 
Figura 1. Número de casos registrados para malária no estado do Piauí nos anos de 2015 a 2019 . Teresina, PI, Brasil.

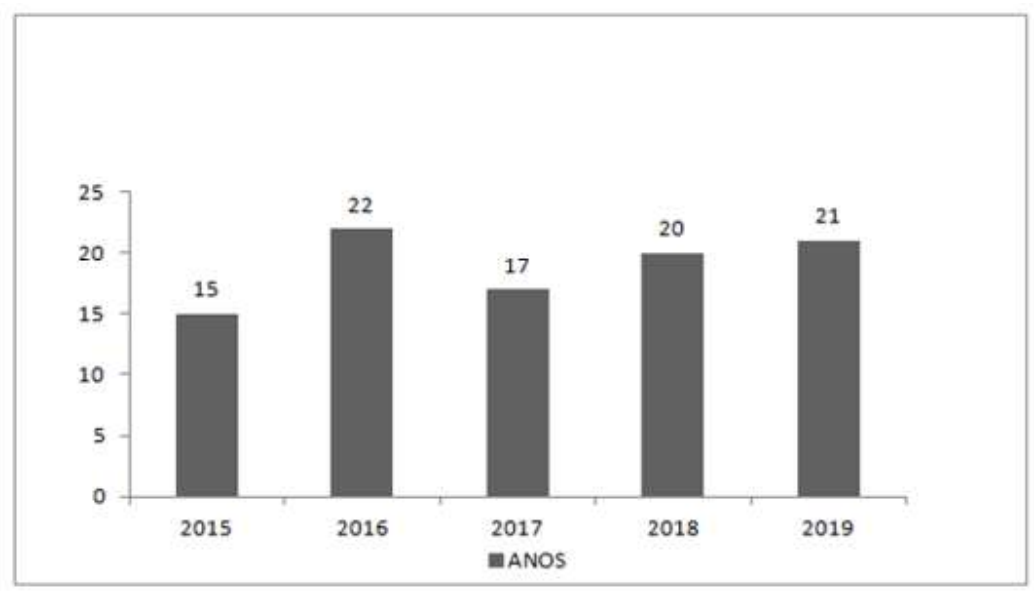

Fonte: Departamento de Informática do SUS (2021).

As cidades piauienses que apresentaram notificações de malária foram Altos, Barras, Campo Largo do Piauí, Itainópolis, Jerumenha, Joca Marques, Luzilândia, Matias Olímpio, Pedro II, Porto e Teresina. Através da análise da Figura 2, foi possível concluir que a cidade de Teresina apresentou o maior número de casos confirmados da doença com a frequência de aproximadamente $42,68 \%$ do total das notificações, com destaque para o ano de 2016, onde obteve-se maior número de registros, seguido por Luzilândia. Por outro lado, os municípios restantes não apresentaram quantidades significativas de notificações ao longo dos anos estudados.

Figura 2. Número de casos por municípios no estado do Piauí nos anos de 2015 a 2019. Teresina, PI, Brasil.

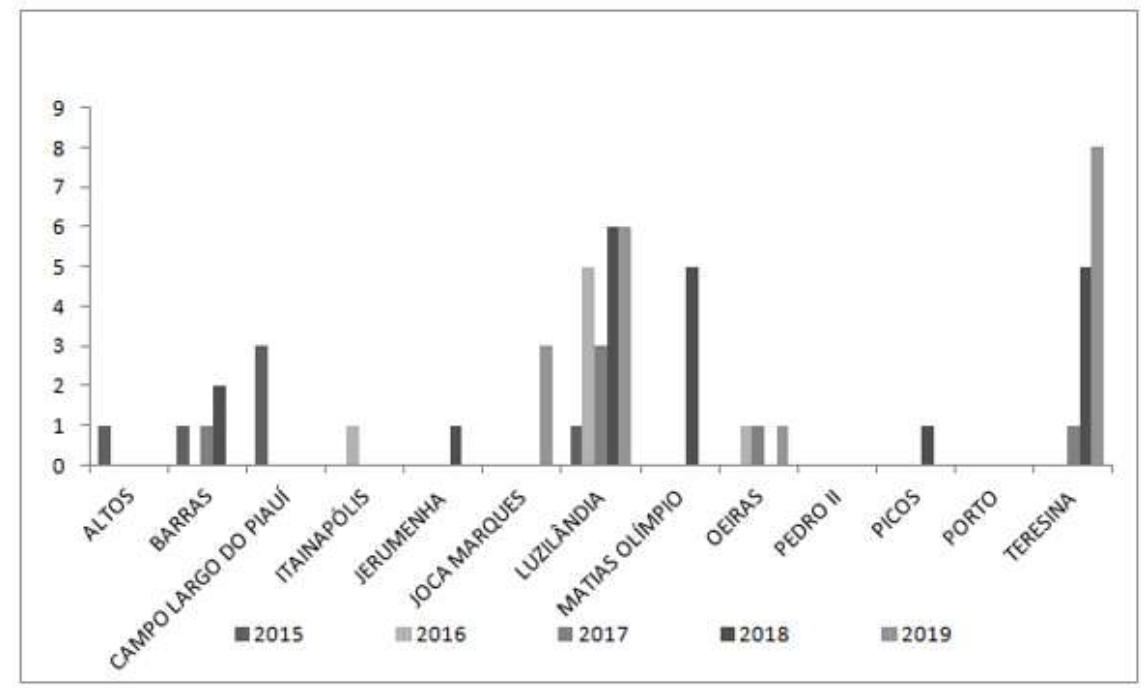

Fonte: Departamento de Informática do SUS (2021).

Quanto a cor/raça, percebe-se através da Figura 3 que a cor parda apresentou predominância nas infecções por malária sendo no total 69 casos, correspondendo a aproximadamente 72,63\% do total. Quanto aos brancos, o grupo apresentou um total de oito $(8,42 \%)$ notificações. Por outro lado, foram registrados sete casos entre os negros o que equivale a aproximadamente 7,37\%. Além disso, a cor amarela representou 5,26\% com apenas cinco casos ao longo desses cinco anos. 
O DATASUS também apresentou informações sobre notificações ignorando a cor/raça, foram informados seis casos, ou seja, aproximadamente $6,32 \%$ da somatória.

Figura 3. Distribuição de casos no estado do Piauí por raça nos anos de 2015 a 2019. Teresina, PI, Brasil.

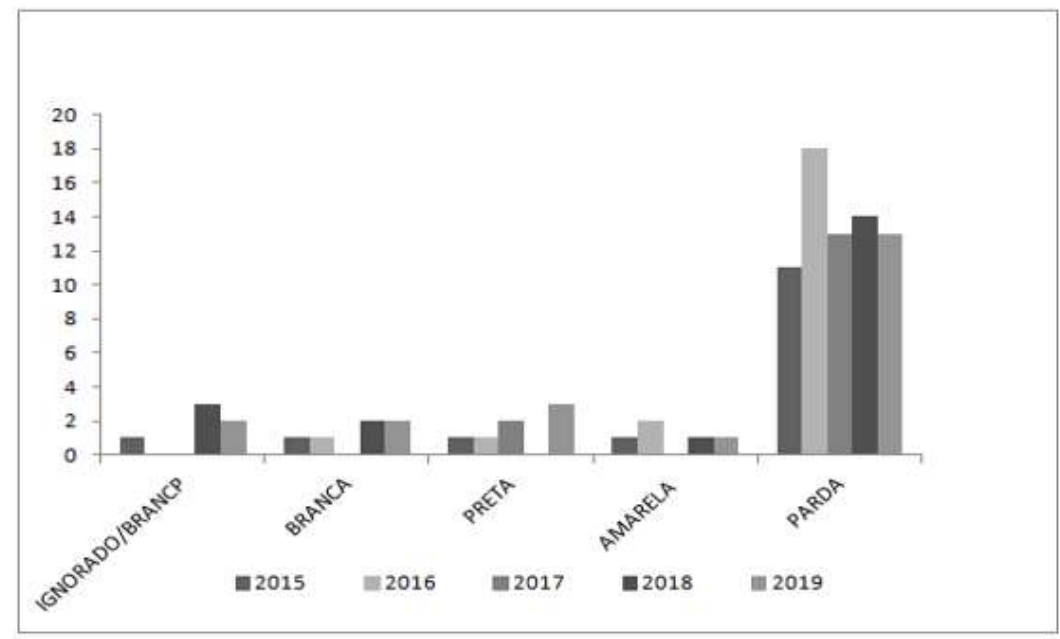

Fonte: Departamento de Informática do SUS (2021).

Observa-se na Figura 4 que houve predominância de notificações no sexo masculino com 70 (73,68\%) casos, além disso, é possível concluir que a faixa etária dos 20 aos 39 anos apresentou altas taxas de infecções quando comparadas as demais. No entanto, no sexo feminino as taxas de infecções apresentam-se baixas $(12,63 \%)$, em que a faixa etária de 40 a 59 anos foi a mais prevalente $(8,42 \%)$.

Figura 4. Distribuição de casos por sexo e faixa etária no estado do Piauí nos anos de 2015 a 2019. Teresina, PI, Brasil.

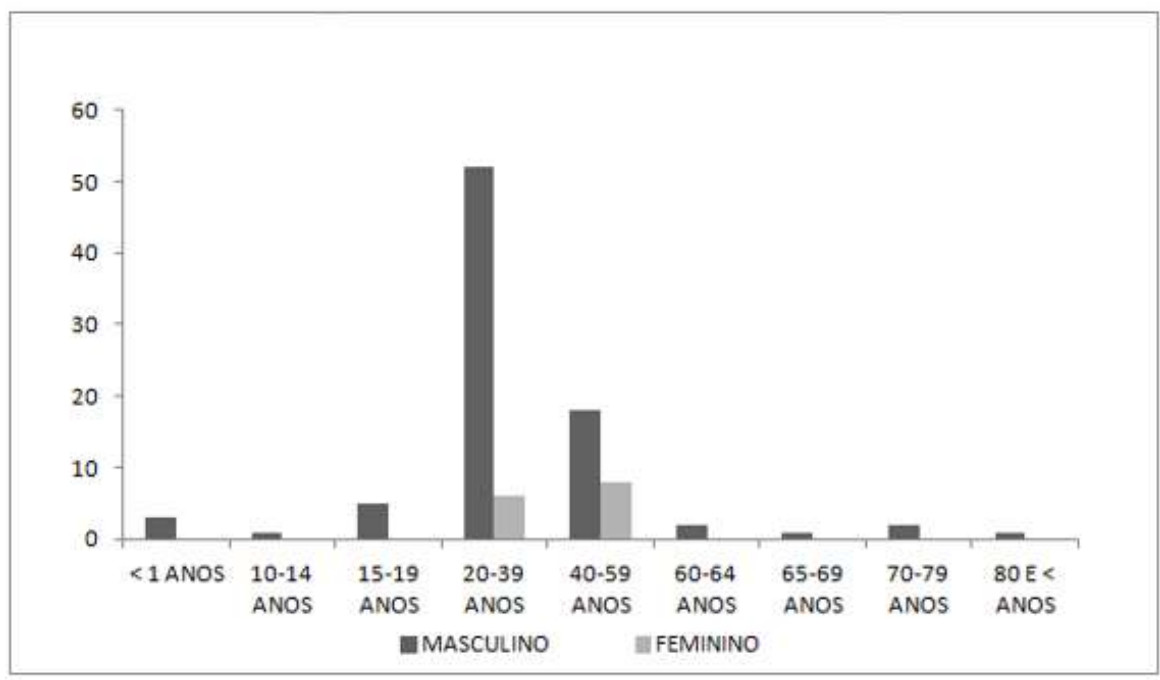

Fonte: Departamento de Informática do SUS (2021).

Quanto a distribuição de espécie de Plasmodium pode-se analisar através da Figura 5 que a maior incidência dos casos predomina a espécie $P$. vivax com 63 (66,32\%) casos, estando presente em todos os anos analisados. Em 2019, foi registrada a maior taxa de casos por $P$. vivax quando comparados aos anos anteriores. 
Nas infecções provocadas por $P$. falciparum foram registradas nove $(9,47 \%)$ ocorrências. Enquanto, por infecções mistas, com associação de $P$. falciparum e o $P$. vivax foram quatro $(4,21 \%)$ casos, $P$. vivax e o gametócitos de $P$. falciparum foram três (3,16\%), P. falciparum e o P. malariae um (1,05\%), e por fim um (1,05\%) caso por P. ovale.

Figura 5. Distribuição de casos da malária, de acordo com a espécie de plasmodium, no período de 2015 a 2019. Teresina, PI, Brasil.

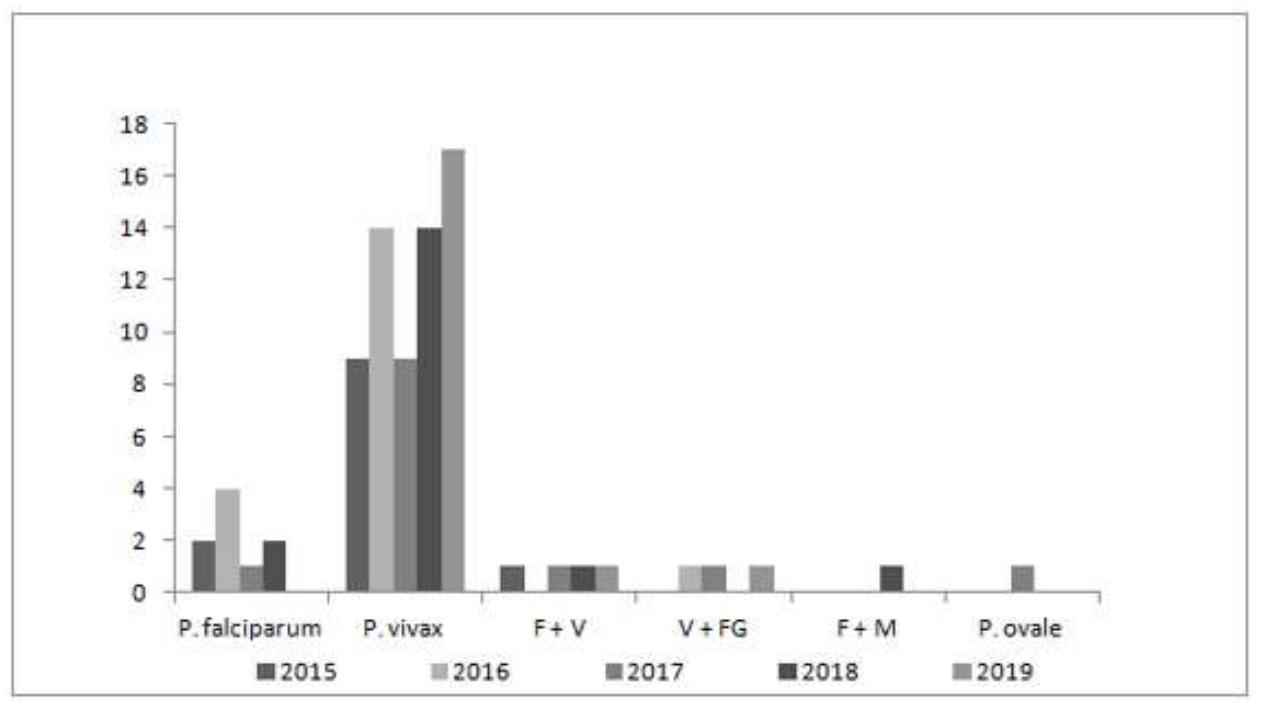

Legenda: $\mathrm{V}+\mathrm{FG}=$ Formas de $P$. vivax + gametócitos de $P$. falciparum $; \mathrm{F}+\mathrm{V}=P$. falciparum $+P$. vivax $; \mathrm{F}+\mathrm{M}=P$. falciparum $+P$. malariae. Fonte: Departamento de Informática do SUS (2021).

\section{Discussão}

Os resultados do estudo apresentam um perfil considerável de características relacionadas a ocorrência da malária no estado do Piauí, apontando que os casos mais frequentes ocorreram entre homens adultos, de raça parda e em decorrência da espécie Plasmodium vivax. Geralmente, isso acontece em decorrência de uma maior exposição ao mosquito vetor da malária, principalmente porque eles se locomovem com frequência nas áreas de garimpo e de desmatamento.

Quanto ao sexo, a predominância que ocorre no sexo masculino pode ser explicada pela atividade laboral, como o garimpo ou o extrativismo vegetal, realizada tanto na região piauiense quanto na região endêmica. Ainda mais, é muito comum em diversos trabalhos sobre a epidemiologia da malária a grande taxa de infecção por indivíduos do sexo masculino. Além disso a faixa etária mais jovem destaca-se no número de infecções por serem indivíduos economicamente ativos (Gomes et al., 2020; Raposo, Santos, Dos Santos, Gonçalves \& Da Silva, 2013; Gonçalves et al., 2020).

Quando comparado os municípios, as cidades de Teresina e Luzilândia, que fazem fronteira com o Maranhão, apresentam um maior número de casos que os outros locais com notificação. Esses casos podem estar associados à emigração de indivíduos portadores da doença para regiões não endêmicas, introduzindo o parasita nessas áreas, visto que o estado do Piauí integra a região extra-amazônica e faz fronteira com Tocantins e com o Maranhão, que fazem parte da Amazônia legal e são áreas endêmicas da malária (Gonçalves et al., 2020).

Outro dado relevante trata-se da comparação das infecções por malária na região Nordeste, o Piauí é o segundo maior estado com notificações da doença. Foram relatados 95 casos, a explicação mais provável é o fato de o estado estar fazendo fronteira com o Maranhão, sendo esta parte da zona endêmica (Do Nascimento, 2015).

A P. vivax é a espécie mais comum nas infecções no estado do Piauí, isso é explicado por essa espécie ser uma das mais comuns em contaminação no mundo todo. Além disso, a própria fisiologia desse parasita permite sua sobrevivência no 
corpo sob a forma de hipnozóita por tempo prolongado, tal fato pode explicar a chegada desse parasita e sua transmissão em uma região não endêmica. Em seguida, o P. falciparum foi notificado, mas com baixa quantidade de casos (Do Nascimento, 2015).

Segundo o censo do Instituo Brasileiro de Geografia e Estatística (IBGE) (2010), a distribuição populacional do Piauí por raça indica que há predominância de pardos totalizando 485.779 pessoas. Esse fato, explica o motivo da ocorrência de casos ser mais prevalente em pardos, quanto maior a população maior a frequência de casos nesta.

Como resposta do governo foi lançado o Programa Nacional de Prevenção e Controle da Malária (PNCM), cujo objetivo é reduzir a transmissão, gravidade e morbimortalidade por malária. O programa foca no diagnóstico e tratamento precoce ofertados gratuitamente, além da prevenção e promoção da saúde (MS, 2017).

Na região extra-amazônica a letalidade da malária é bem maior, principalmente por causa do diagnóstico tardio. Isso ocorre devido à pouca habilidade dos profissionais da saúde em áreas não endêmicas. Além disso, os sintomas são inespecíficos e comuns de síndromes gripais, o que pode confundir os profissionais causando atraso no diagnóstico e, consequentemente resultar em doença grave e óbito (De Pina et al., 2010).

Apesar do Piauí ser considerado uma região não endêmica, devido a movimentação populacional pela Amazônia legal, aumenta os riscos de transmissão no estado, tornando-o mais suscetível ao aparecimento de surtos. Dessa forma, é necessário que a vigilância epidemiológica desenvolva suas atividades constantemente com técnicas de prevenção e controle da doença, associado ao diagnóstico rápido para obter um tratamento precoce e eficiente (Braz et al., 2020).

\section{Conclusão}

Este estudo avaliou um total de 95 casos de malária no estado do Piauí. Houve prevalência de notificações no ano de 2016 em que a cidade de Teresina apresentou o maior número de casos confirmados, sendo os mais atingidos os indivíduos do sexo masculino, cor parda, com idade entre 20 a 39 anos. Quanto a distribuição de espécie de Plasmodium pode-se analisar maior incidência da espécie $P$. vivax.

Nessa perspectiva, conclui-se que a malária é pouco comum no Piauí e que a maioria dos seus casos notificados provém de outros estados. Dessa forma, é importante destacar que as equipes de saúde e de vigilância epidemiológica mantenham sempre alertas sobre a presença dessa doença no Estado, para oferecer tratamento precoce e melhoria no prognóstico dos enfermos, a fim de evitar que o vetor transmissor seja infectado e consequentemente impedir a formação de uma cadeia de transmissão.

Almeja-se que este estudo possibilite aos profissionais de diversas áreas conhecer mais sobre a distribuição dos casos de malária no Piauí, e sobre como este problema encontra-se atualmente no estado, bem como desenvolver o senso crítico para criação e implementação de estratégias eficazes e confiáveis para reduzir o número de casos, bem como orientar a população acerca da doença e sua gravidade.

\section{Referências}

Braz, A. R. P., Bringel, K. K. M. C., Oliveira, L. A. P., Oliveira Filho, I. F. C., de, Trajano, I. L. O., Costa Júnior, . . . \& De Oliveira, B. L. C. A. (2020). Caracterização dos casos de malária na região extra-Amazônica brasileira entre 2012 a 2017. Journal of Management \& Primary Health Care 12(5), e5.

De Pina, C. A., Da Silveira, B. C., Pedro, R.S., Valls-de-Souza R., Da Silva, S., De Souza, P.R., . . \& Brasil, P. (2010). Diagnóstico tardio de malária em área endêmica de dengue na extra-Amazônia brasileira: experiência recente de uma unidade sentinela no Estado do Rio de Janeiro. Revista da Sociedade Brasileira de Medicina Tropical, 43(5), 571-574.

Do Nascimento, J. A. A. (2015). Epidemiologia descritiva da malária no Estado do Piauí, 2002 a 2013 (Dissertação de mestrado). Fundação Osvaldo Cruz, Teresina, PI, Brasil. 
Research, Society and Development, v. 10, n. 5, e47110515180, 2021

(CC BY 4.0) | ISSN 2525-3409 | DOI: http://dx.doi.org/10.33448/rsd-v10i5.15180

Fonseca I. E. F. B. (2017). Estudo da malária com relação aos aspectos de clínica, diagnósticos e tratamentos (Trabalho de Conclusão de Curso). Instituto Brasileiro de Medicina e Reabilitação, Rio de Janeiro, RJ, Brasil.

Gomes, M. S. M., Menezes, R. A. O., Vieira, J. L. F., Mendes, A. M., Silva G. V., Peiter P. C., . . \& Machado R. L. D. (2020). Malária na fronteira do Brasil com a Guiana Francesa: a influência dos determinantes sociais e ambientais da saúde na permanência da doença. Revista Epidemiologia e Serviços de Saúde, 29 (2), e181046.

Gonçalves, C. W. B., Rodrigues, R. A., Neto, A. B. P., Gomes, D. L. F., Silva M., \& Sorte G. V. B. (2020). Análise dos Aspectos Epidemiológicos da Malária na região Nordeste do Brasil. Revista Amazônia Science \& Health, 8(2), 42-50.

Instituto Brasileiro de Geografia e Estatística. (2010). Censo da raça no estado do Piauí [Internet]. Recuperado de https://cidades.ibge.gov.br/brasil/pi/teresina/pesquisa/23/25359?localidade2=22.

Ministério da Saúde (2019). Malária: o que é, causas, sintomas, tratamento, diagnóstico e prevenção [Internet]. Recuperado de https://antigo.saude.gov.br/saude-de-a-z/malaria.

Ministério da Saúde (2020). Guia de tratamento da malária no Brasil. Brasília: Ministério da Saúde.

Ministério da Saúde. (2010). Guia prático de tratamento da malária no Brasil. Brasília: Ministério da Saúde.

Ministério da Saúde. (2017). Programa Nacional de Prevenção e Controle da Malária- PNCM. Brasília: Ministério da Saúde.

Raposo, C. C. B. S., Santos, J. B., Dos Santos, G. M. C. Gonçalves, E. D. R., Da Silva, A. R. (2013). Plasmodium vivax malaria: related factors to severity in the State of Maranhão, Brazil. Revista da Sociedade Brasileira de Medicina Tropical, 46(1), 67-72.

Secretaria de Saúde do Estado do Piauí. (2017). Situação epidemiológica da malária no Piauí: nota informativa. Teresina: SESAPI 